\title{
Index to Volume 11
}

Abbassi, V. 8012

Ahramson, D. 802

Aborption 972, 1033

Achord, D. 810

Acidosis 1177

Actinomycin 1) 282

Adams, A. J. 770, 840

Adanı, F. H. 178

Adenine phosphoribonyltramserase (APRI) 69) 1

Adenosine $33^{\prime}, 5^{\prime}$-monophosphate (cyclic AMP) 3.3

$S$-Adenosyl methionine decarboxylase 180

Adolescence 88.3

Adrenaline 889

Adrenocorticoid insufficiency 770

Aintender, I: 808

Alagille. D. 994

Alanine 720.943

Albumin 37.163 .167

Aldosterone 182,8.37

Alexiou, D. 6th

Altax, $C .147$

Alternative pathwaly 285.910

Alveolar macrophages 197, 12012, 12118

Amino acids 720. 804.972

4-Amino-5-imidazolecarboxamide (AIC A) 69)

5-Amisno-1-ribosyl-4-imidizolecarboxamide (rAICA) 69!

Amniotic fluid 119, 878, 894, 1057, 1072, 11.37

Anderson. H. (. 920)

Anemia 7.58

Angiotensin 8.37

Ansell. C. 1177

Antidiuretic hormone 3.3

Antigen 1129

Aperia. A. 1109

Apgar score $7+4$

Apnea 709.791

Appropriate for gestational age $(A(B)$ infants 108

Archic. J. P.. Jr. 867

Arginatse 949

Argininosuccinate synthetase 949

Armstrong. R. 13.5

Arnaud, P. 986

Arylsulfatase A 1072

Asbrock. T. 285

Asparate transcarbamylase 29.3

Asphyxia 889

Asthma 732

Atkin. C. 1.. 82.3

ATPase 153

Auricchio. \$. 997

Bacteriat 11.33

Bactericidal activity 1208

Baker, S. 1102

Baldwin. M. R. 744

Bansil. E. 681
Barlow, B. 9() 6

Barrett, C. T. $X+5$

Barric. H. 1177

Bittc. S. P. 11+4

Bcilmear. P. 71

Beicr, 1. 1045

Bellanti, J. A. 1. 158, 197, 224, 10015. 1202.1208

Belshe. R. B. 204

Benke. P. J. 936

Beram. A. 1'.779

Beratis. N. (i. S62

Berger, M1 3.3

Berline (i.11.112

Bernasconi. Mr. V. 10+2

Beutner, K. R. 2.31

Bicarthenate (ot)

Bickel, H. lost

Bidlingmaicr. F. (1)

Bile acids 11 so

Bilirubin 16.3.167, 171, 8018, 8801. 106.3

Bilirubin hinding sos

Biotin $11+4$

Bistoletti. P. sist

Bjorkstin. B. 910

Blickhum. W. R. 770

Blakely. (i. A. 1051

Blattiner, R. J. 6.5

Bleod 744.754.871.878

Blood cord 135

Bloced wolume sis

Blumenfeld. T. A. 1100.3

Buat. I. F, 898, 977

Bogarl, B. I. 1.31

Boichis. 11. 1124

Boivin. P. 271

Bonta. B. W. 754

Borkelman. H. 108s

Bonchut. R. B. 276, 79)

Bower ( . 271

Brain 28. 2901. 1042.1198

Brain development 104?

Bratin glscinc 1192

Bras P. F. 823

Breathing 24.967

Brem S . 939

Broberger. (). $11(10)$

Bronchial asthma 14.3

Brot. F. Sith

Brown, LE (i. seste

Bruce-lagoc, A. A. $1+7$

Bruinvis. L. I08x

Buchler. B. 186

Buncher. ('. R. 13

Burmeister. 1.. (ot)

Burrous, B. 218

Busse. ( $7(10)$

Butconandt. ().91. 885

(3. (3) 285

Calcium 112, 739, $8.37,1180$

(alcitonin 112,1180 (alcium homeontasis 7.39)

(alcium ionophore 1.31

(allihan. J. W. 1100

Carbohudrates 7201. 8014

(arbonic anhedrase 11134

Cardiate output 867

(arnitine 878

Carvajal. H. 1. 33

Canhore. W. J. 967

(a)tell, S. Y) 21

(antenadia. A. 1117

(atch-up growth 6os

(attecholamines sise)

(cillerowth 180

(c)ll surfiace 76,5

(crebcllum 293

corchral cortex 298. 655

Cerchrompinal fluid 37

Chance (i. W . 874

(hang. J. P. 7hs

(hang. 1.. ().

(hang. 1.-11.37

chancict. R. .11. 26t

(hemotaxis 077.732

(herry. J. 1). $250.9(14$

(berkin. H. 106.3

(holesterol 19. 744. 453

(holine kinase $8+4$ )

(holinephouphotransterane 770

(hopard. J. P. 1/80)

Chronic granulomatous disciase (c(il) 1.58

chronic lactic acidosis 1198

(iilia $)(1) .4$

(iliary dykine ia 14.3

Ciliars dekinesia factor $+5,1.31$

(lark. B. R. 2()3

Clouticr. A. 116

(lyde. W. A.. Jr. 2.34. 24.3

(icenasme protection 100

cold ciponure 79.3

(vile. M1. P. 7.37

(i)llicr. A. .11.234.243

colestrum 1177

complement 285.077.910

(omplement fixation 1124

(omposition 701

Concinavalin $\mathrm{A} 76.5$

conderclli.s. 24

conforti. A. 112

Congenital abnormalities 10.3

conod. F. J. +5.131

Comover. J. 11. 45.131

Continuous positive airway pressure 967

Copper 823.103.3

(orde serat 739

coronars bloced flow 867

(orrigan. J. J.J.Jr. 910

Cortivel st.5

Comem. (i. 758

(irmi. F. L. 24

(intelex. K. 1171

colton. R. (i. H. 6.31 
Cottrcau. D. 271.1123

Cousins, I. S94

Cowell, R. M. 1162

Cow: milk proteins 898

Crawhall, J. C. +1

(reatine 880

Creatinine 880,1129

Cress. H. R. 19

Crestcil. 1. 720

Criswell. B. S. 71

Cutaneous stimulation 24

('ut\%. E. 279

Cystathionine $\beta$-synthase 100

Cysteine. methionine 28

Cystic tibrosis 45, 131, 186, 765, 827.981. 1133,1148

Cystic fibrosis protein 14.3

Cystine 41.685

Cystine storage 124

Cystinosis 41

Cystinosis, infantile nephropathic 124

Cytidine-5'-diphosphocholine 849

Dahlmann. N. 695

Danks, D). M. 6.31

Dar. H. 103

Darby. B. 180

Das. M. 1068

Dauncey. M. J. 10.3.3

David. I. 1180

Daviduon. R. G. 1072

Davis. (S. W. (C. 174

de Bree. P. K. 1088

De Fazio. S. R. 71

Degenhart. H. J. 1047

de Groot. C. J. 1112

Delannoy. ( . W. 298

DelValle, J. A. 2

Delvin. E. 124

Denns.F. W. 234

DePape-Brigger, 1). 124

de Romaña. (j. L. 774

Desmond. M. 82

Developmental biochemistry 293

Dexamethasone 705

Diabetes insipidus, nephrogenic 33

Diamond. I. 758

Di Donato. S. 1094

Diet 182.804

Difference spectroseopy 16.3

Differentiation 197

Dihydroliponl dehydrogenate 1198

Dilution curves osil

Dipeptide 972

Diptheria 1211

Discontinuous density gradient 11.59

Disseminated intrataiscular coagulation 916

Dithiothroitol 41.124

DNA 3015.796. 929.939

Doberstyn. F. B. 158

Dresmant (i, R. 71

Dubois. J. D. 116

Dudley. J. P. 904

Dussialt. J. H. 116

Dysmaturitt 8401

I:cema 7.32

Iidmunds, 1). 1106

I:dson. J. 1. .793

Effective clastance 791
Effective pulmonary capillary blood flow 1171

Eichhorn, W. 704

Electrolytes 1177

Electron microscopy 765

Electrophoresis 808.1072 .1148

Eller, J. J. 247

Ellis. F. F. 227, 26,3

Ellsworth. D. 1.. 0.55

Embryo, chick 305

Endethelial permeatrility os

Endotoxin 916

Energy homeostasis 962

Encrgy metatholism 2901. 298

Enterocolitis 9(0)

Enterolinase activity 282

Environment 695

Enzymatic induction, in utero 282

Enzyme activation $[00$

Finzome replacement 816

benzme uptake 811.816

Enzyme differentiation 6069

Enzymoimmunodiffusion 1072

Fnamoimmunocketrophoresis 1072

Epithelial cells 9.36

Epithelial lung cells 305

Erhe, R. W. 1137.1141

Erlanger. B. F. 939

Erythrocytes 16.3, 167.271. 691.115.5

Erythropoiesis 9. 758

Estrogen. estrone 9)

Exeni. R. A. 153

Fxperimental allergic encephalitis 37

Extrauterine life 950

Factor B 9ll;

Fallat, R. W. 13

Falletta. J. M. 78

Familial hypertrigleceridemia 13,953

Fanaroff. A. A. 977

Iatrell. P. N1.770, 840.1165

Fat absorption 774

Fat digestion 11 son

Faull. K. F. 6.31

Fecdback control s8ul

Ficig. S. A. 1068

Foldman. B. H. $71+$

Fordinandus, I. D. . sot

Fernbach. D. J. 78

Fetal 627

Fetal growth losi

Fetur 2, 6. 19. 24, 95, 116. 271.649 .655$. 6(69). 758. 770, $8+0,8+5,85 x, 87 x, 889$. $1045,1051,1057,1155$

Jibroblasts +1. 186. 641. 685. 749. 811. $\times 62.981 .1137 .11+1$

Fishel. ( . W . 1.35

Fisher. D. A. 627.833 .837 .962

Fisher, 1). 1: 248

Fitzgerald J. F. 8014

Fixler. 1). 1. 867

Hax. N1. H. 37

Feisher. 1.. D. 100

Fluharty. A. 1.. 894

Follicle-stimulating hormone (FSH) 91

Forristit. J. 285

forsgren. A. 910

Frankel. H. A. 811

Fratske. M1. 1. 972

freedmin, D) A. 85

freman. R. 6.31
Frem. I). 13.823

Fricdmatn. W. F. 190

Frohlich. J. 878

(1-1-Fuconidase 862

Fucosidesis sto?

Fudenberg. H. H. 139.143, 317,980

Funkenstein. B. 112

Furesemide 8.37

(iacrlan. P. 45

(iathr, M. 4

(iatactokinase 1045

(ialactose-1-phosphate 1045

(ialactosyl transferase 981

(iitll, D). (i. 274)

(ian. 1. 6.31

Giardner. P. S. 254

Garvin, J. H.. Jr. 1159

Gas chromatography 6.31

(ialucher's discase $6+1$

(iaull. (i. F. 28. 100)

Gawron, E. R. 7.54

Gazit. H. 1129

(icl filtrattion $7+4$

(ienctic discase $1+3$

cienctic disorder 1123

Gestattional alge 791.871.959

Gilbert. 11.95

Gilden. (C. 845

(iiv)la. I. A. 1042

(iirard. F. 182

Glaser. J. H. 811

(ilasgow, A, M. 30).3

(ilezen. W. P. 234

(ilobin chain synthesis 9

Glomerular filtration rate 959

(ilucigen 108. 664. 94.3.962

(ilucatric acid siso

(iluck. I. 71t

(ilucocorticoid $8+0$. 1012

(ilacocorticoid recepter protein 705

(iluconeogenesis $95,94.3$

(ilucose 95. 108. 153.298. 845.874.962

(ilucose-6-phorphattase 936

Cilucose-fi-phosphate dehydrogenase ( $(i-6)$ PI) 1.58

Cilucose phosphate isomerase atctivity 1123

Gilucose tolerance 1.3

$\beta$-Cilucosidase $6+1$

$\beta$-Glucuronidase 811,810

Giluech. (. J. 13.3.95.3.957

(ilutamate 7?0)

Glutathione reductiose 685

(ilycerol 95. 298

[1-140](ilycerol 95

(ilycinc 1155

(ilycine dealvage enzyme system 1192

(ilycine encephalopathy 1192

(ilycogen $8+10.845$

Glycogen storage diseare. lype I tot

(ilycogenolysis 94.3

(iodfrey. S. 1171

(ioldman, H. 124

(ionadotropin 91

(ionzalez-Noricgat. A 816

(iritmeyer. D). ( . 1180

(irithatll, (i. (i. 774

(irajwer. 1.. 962

Grajuer. I.. A. 8.33

Gravely. M. 147

(ireengard, () 2. 660) 
(iribble. T. J. 1106

Grimani. M. 646

Gimanis. A. P. 640

(irimes. E. 677

(irowth 112.695

Growth hormone $8 \times 5.1180$

Guancial. R. I. 1155

Haddial. J. (i. 7.39)

Hageman factor (coagulation factor $X I 1) 910$

Hahn, P. 878

Hakimson, D. (). 967

Hall. ('. B. 235

Hallman, M1.714

Hallock, J. A. 135

Halpern, B. 6.31

Hammond. J. 6.31

Hansen. S. 1192

Harris. D. 685

Harvey, D. R. 723

Hashemi. S. B. 420

Heart 48.174.1198

Heitrt rate 889

Hechtman, P. 1(1)39

Heim. 1.. R. 71

Heiner, D. (. 119

Heird. W. ( . . 906

Hemoglobin Barts 147

Hemoglobin $\alpha$ chain deficiency $1+7$

Hemolytic discise ()

Henderson, F. W'. 234

Herin. P. I 104

Herpes simplex virus encephalitis 37

Herschkowitz. N. 1094

Hasterberg. I. 178

High density lipenpotein cholenterol (c) I.DI.) 957

Hill, D. I. 874

Hill, D. W. 1171

Hillman, 1. S. 734

Hilton, H: N. 67

Hirschhorn, K. 862?

Hirschklatu. N. J. 190

HI.A-B12 7.32

Hohel ( . J.119

Hodes. M. 1: 13\%. 1148

Hodson. W. A. 10151.10157

Hoffman. J. I. I: 867

Holland, W'. P. J. 1097

Hom. D. 76.5

Homeostasis 874

Homes, 1. A. 1112

Homocit rullinurial 949

Homocesteine 11.37

Homocystinuria 100

Horkovit. A. $880(1)$

Huamg. ( . . 1. L. So

Hughes. W. T. 305

Huisman, I. H. J. 147, 1102

Hull. 1). 79.3, 11+4

Hulstilert. ( . : : 1112

lluman enthrocstic carbonic anhwdrase 871

Human milk 1177

Hurley, I... 758

Huxtable. R. F. 774

Hedrocortisonc 282

$25-H$ dydroxy-vitamin D) 7.34

Honic. I. 878

Hyperammonemiat 949

Hyperbiliruhinemia 9.39

Hyperlipoproteinemia 19)
Hyperlysincmia 949

Hyperuricemia 6ot

Hyperventilition 967

Hypoglecemia 279,840, 94.3

Hyponatremia 1177

Hypoplasiat 920

Hypoxanthine-guanine phosporibouyltramsferase (HAPRI) (6)

Idiopatthic nephrotic syndrome 910

Idiopathic precocious puberty 91

Idiopathic hort stature $8 \times 5$

$\lg (i$ suhclasse- 1211

Ikegami. N1.178

Im. S. 6.31

Immune wstem 1.35

Immunoglobulin ( $; 37.45$

Immunoreactive glucigen 8.3 .3

Impedance caldedographa 1171

Infant nutrition 774

Inorganic phomphate ont

Inosine 5'-phosphatte (INIP) 69)

In situ hrbridization 929

Inulin 1.3. 108. 83.3 .94 .3 .962

Intestinal absorption 1.53

Intestinal development 804

Intralipid 17 I

Intratuterine growth retardation $8+0$

Inulin 28.5

Inulin clearance 959

locelectric focusing 1+3,11+8

Formames 871

Inoproteremol $\$ 27$

Istorme 1072.1150

Jatcots. J. ( . 7.32

Jatme. I. 147

Jitundice 171.1063

Jentsch. I: 1)

Kithn, A. 271.1123

Kalvi. H. K. 290

Kang. I. S. 655

Kaplan. B. S. 1039

Kiolian. S. A 845

Karpowice. K. 19

Kashyalp. M. L. 953

a-Ketoglutarate dehydrogenase 1198

ketone hodien 29)

ketting. D. $108 x$

Kidnes 124.182.049.1129.1198

Kihara. H. 894

Kim. H. W. $26 t$

Kim. Y. 910

Kinky hair sydrome 82.3

Kirkpatrich, 1:714

Kitagituat. I. (1)+1

klein. W. 11168

Kleinerman, J.1.977

Kimoller. N1.920

Knorr. 1). 91, 885

knowles. H. (...Jr. 13

Knudson. R. J. 218

kocnigsberger, M. R. 106,3

Kogut. N1. 1). 60,

Konotey-Ahulu, F, I, D, 147

Koumantakis. 1: (1)

Kretchmer. N g92

Krieger, 1. 790

Kropp. K. 67

Kupffer cells $6+1$
Kusumoki, I 728

I.ahadic. ( 3,862

I abal. .11. I. . 11,33

lactate $29 x .1198$

I atfin. R. 19)

l.atgercrante. H. 884

latm. H. 1102

I.amellar bodien sty

lamcllar meclin 197

L andian. S. A. 1155

l.ead poisoning 153

I. cukc, R. D). 959.962

l.ebenthal. 1. 282

I chlitnc D) 1036

I.chouite. J. 749

l.chowitz. .1. D. 218

l.ecithin 714.723 .1057

I.e. J. A. 744

I.ce Y.J. Sor

lenfint. ('. J. 204

length 7011

I. roux. J.-P. 720

I.csch- Nyhan sndrome (6)1

I.cuhecres +1.124 .158$

l.eukecytes, polymorphonuckear 1.35

l.cung. $A .1160$

levine, (). R. 6si

I.witsky. I. I. 298

l.ifhity. $\mathrm{F} .153$

I.inderkamp. (). 885

lipis 167.174.744

I ipickese ot 1

lipolsis 94.3

l.ipoproteins 19.74t

I.ipp, K. 705

litwin. S. 1). 1211

liver 2, 6, 28, 124, 174, 279, 3113, 6.31 $6+1.646 .669 .840 .1045 .1137 .1198$

L.ombardi. N. 37

Longhi. R. ( .100

I.orent/. W: B.. Jt. 3.3

l.ou hirth ucight intant 10.3 .3 .1180

low density lipoprotein cholenterol (c) HIDI.) 957

lone. C. . . . 1079

l.141. B. A. 69)

L./S ratio 1057

I.ubit, B. W. 9.39

l.ucis, D) () 233

I. uit-1)c Hatan. (i. 1112

1.ung 178. 305. 681.723 .770 .845 .849 . 858.11151 .1057

l.ung surfictiont 114

1.ung volume 79 )

I.utcinizing hormone (1.1) 91

l.smphoblasts 11.37

l. rouphospholipase 858

l. rsosomal enzymes 12(1)

l. visesomal storage disease 862

linoromes 197.811,816. 1202

l.inozyme 916.977

Matckler, B. 1.71

Macl cein. W. C...Jr. 774

Mader. T. 8R5

Magnesium 112,7.39, 1033, 1180

Magnesium deficience 758

Matibath. H. 1. 7.37

Malformations 758

Malnutrition 17 ! 
Mamer. O. 124

Mamer. O. A. 1192

Manniello, R. L. 840, 1165

Manz, F. lo8t

Marie, J. 271

Marrec, D. 182

Martin. H. L. 174

Martinez, J. R. 827

Mass spectrometry 6.31

Mastromarino, A. 67

Maternal nutrition 804

Mathewson. J. W. 48

Maturation 845,1208

Maver, A. M. 10008

McCance, R. A. 1081

McConnell, R. F.. Jr. 3.3

McGregor. S. M. 141

McLean. R. H. 910

McVicar. M. 153

Mead, J. F. 174

Mehta. S. 290)

Mellies, M. J. 13.953.957

Mellins. R. B. 268

Membrane fluidity 765

Menkes. J. H. 290

Merchant, K. 802

Merritt, A. D. 138, 1148

Metabolic rate 796

Metabolic responses 79.3

Metabolism 874

Metachromatic leukodystrophy 1072

Methylcitric acid $11+4$

3-Methylerotonylglycinuria 1144

Methylenetetrahydrofolate reductase 1137

Methylenetetrahydrofolate reductase deficiency $11+1$

Methylmalonic acidemia 749

Methylmalonyl-CoA mutase $7+9$

Mettau. J. W. 1097

Michacl. A. F. 910

Milk 28

Milk leukocytes 906

Mills, M. 10.39

Mitochondria 6. 279. 30.3

Mohapatra.S. N. 1171

Molecular hybridization 929

Molish. B. 82

Monin. P. J. 967

Monnens, L. A. H. 108x

Monocyles 677

Mononuclear cells lo6s

Mononuclear phagocyles 906

Monsher, M. T. 139. 14.3

Montgomery. J. R. 6.3.65.67.71.87

Moore, A. 1177

Morag. A. 231

Morgan T. E. 10151.1057

Morikauat. Y. 728

Morris M. D. 744

Norrow, G., Ill 749

Motoneurone inhibition 709

Motoyama. E. K. 220

Moynihan. J. B. 871

Mucolytic agent $9(1) 4$

Mucous glycoprotein 977

Mukhopadhyaly. N. 71

Murphy. J. H. 1051.1057

Murphy. M. 82

Murphy, M. J.. Jr. 305

Muscle atrophy y20

Musher K. K. 84
Mutation 1123,1133,1141

Myocardial blood flow 867

Myristic acid 723

Nadas, A. S. 1117

Nadler. H. L. 981

Naftolin. F. 701

Nain, (. K. 290

Nakamura, T. 728

Nakao. T. 949

Nathenson, G. 1211

Natural surfactant 178

Neese, A. I. 783

Negishi, H. 936

Neonatal development $8(14$

Neonate 135, 276, 298, 723, 754, 791,874. $878,906,939,957,962,106.3,1171$

Nephropathic cystinosis 685

Nerurkar. L. S. 197, 1202,1208

Neurohypophysis 6.27

Neutrophil 135.677.732

Newborn 9, 19, 28, 48, 10,3, 147, 171, 182. $646,649,677,720,737,793,833,858$. $871,880,916,1033,1068,1155$

Newborn infant 116?

Nichols. B. 1. 80

Nikethamide 880

Nitowsky. H. M. 1013

Nitroblue tetrazolium 1.58

Nitrous oxide 1171

Nonhepatic tyrosinemia 6.31

Nonketotic hyperglycinemia 749.1192

Noonan. P. K. 7.37

Noradrenaline 889

Norman. M. 1:. 7.32

Nowak, M. 878

Nozaki. M. 178

Nucleic acid synthesis 293

Nucleotide hodies 939

Nutrition 695. 796

Nyhan. W. I. II 14

Obesity 13

Ogra, P. I.. 2.31

Ogra. S. S. 231

Oh. W. 967.1162

O'Neill, P. 71

Opsonization 910

Organ culture $9(44.977$

Ornithine methionine decarboxylase 186

Ornithine transcarbamylase 3013

Osang. M. 1045

Oshima, R. G. +1

Osteolysis. epiphyseal yב0

Oxada. M. $6+1$

Oxidative enzymes 1202

Oyanagi, K. 949)

Dyasceter. S. 94.3

Pagel. (. 9)16

Pahua. R. 677

Pahua, S. Ci. 677

Palmer. S. 1051, 1057

Patmar crease 103

Pancreas 943

Papadatos, ( . 6to

Papatiangelou. (i. 646

Paper chromatoclectrophoresis ser

Paramyxovirus 174

Parathyroid hormonc 112,739,1180

Parkinoon, C. F. 72.3
Parrott, R. H. 264

Paton, J. B. 298

Pelger. A. 171

Penn-Walker. D. 878

Pentenoic acid 303

Percutancous absorption 7.37

Peroxidase staining 9.39

Perry, T. I. 1192

Petersen, K. 695

pli 1177

Phagocytes 677

Phagocytic uptate 1208

Phagocytosis 9(06. 910

Pham-Hut-Trung, M. T. 182

Pharmacokinetics 7.37

Phenobarbital 880

Phenylatanine hydroxylatse 2

Phosphatidylcholine 770. 845

Phosphatidylcholine-lysophosphatidylcho-

line cycle $8+9.8 .58$

Phosphatidyl dimethylethanolamine 72.3

Phosphodiesterase 655

Phosphofructokinase 1159

Phosphofructokinase, erythrocyte 271

Phosphoglycerate kinase 1068

Phospholipids 723. 1202

Phospholipids, acidic 714

5'-Phosphoribosyl-5-aminoimidazole-4-car-

boxamide (AICAR) 69!

Phosphorus 1180

Phosphorylcholine cytidyl transferase 8.58

Phototherapy 9.39.106.3

Phytohemagglutinin lots

Picou, D. 6.37

Pifer. 1.. L. 30.5

Pitt, J. 906

Pituitary 627

Pivettat O. H. 11.33

Placenta 95. 646.894

Placental transter 116

Plasmia 8.3.3, 88.3

Plasma membranes 174

Pneumocystis carinii 305

Pojerovit. A. 880

Poley, J, R. 1180

Polmar. S. H. Sus

Postnatal period 720

Potts, F. 80

Preunisone 1042

Pregnancy $646,972,1042$

Premature infants 108.754

Prematurity 874

Prenatal diagnosis 936. 1072

Pressure-volume characteristics $17 x$

Preterm infant 709. 8012

Primate 1051

Proctor, K. (i. 779

Properdin 285

Propionyl-CoA carboxylase 749,1144

Pross. S. H. 1.35

Protease deficiency 981

Protein 796. 827.972

Protein biosynthesis 720

Protein-caloric malnutrition 290

Protein deficiency 972

Protein intake 774

Prucitt. J. 1.. 10.57

Pryse-Davies, J. 723

Puberty 701

Pulmonary hemosiderosis 898

Pulmonary stenosis 867 
Pulmonary surfactant 714

Pyrimidine biosynthesis 203

Pyruvate 943

Pyruvate carboxvlase 279, 6.37

Pynuate carhoxylade activity 6

Pyrubate dehydrogenalse 279, 6.37, 119

Pyruvalte kinase 1068.1159

Quic. P. (i. 910

Rabbit tracheal hioassaty 1.31

Rithbit tracheal epithelium 14.3

Rabc, 1:. 1:. 37

Rabinovitch, M. 1117

Radioactive microspheres 867

Radionllergosorbent tests (RASI) 898

Rato. (i. J.S. S8

Rilssin, D) K. 28

Ritt 6

Rat liver 749

Rattazzi. M1. ('. 1072

Raux-turin, M. ( . 182

Red hlood cell 10139

Red cell mass 885

Reese. A. 1102

Reid. L. 210

REM sleep $7(1)$

Renal function 1162

Renal tuhular acidosis 10134

Renaud, H. 1180

Renin 8.37

Rennert. (). M. $18 \%$

Reserpine 827

Resistive loading 270

Respiration 276

Respiratory distress 1162

Respiratory distress syndrome 119.714. 754.802

Respiratory system 791

Restricted antibodies 1211

Revin, B. 749

Reye's sydrome 279.6.37

Ribosomial genes 924

Richardson, I. S. 264

Richic, E. 71

Ricgel. K. P. 88.5

Riemenschncider, I. A. 48

Rigatto, H. 276.791

Right ventricular hypertrophy 867

Ringelhann, B. 147

RNA 30.5. 929

RNA synthesis 705

Rohillard. J. F. 6+9

Ruhionsen, 13. 11. 274, 1198

Robinson, H. 6.37

Roc, I. F. 604

Rojanasathit. S. 7.39

Romano, M. ('. 1042

Rose, J. 920

Rosenblatt, D) S. 11.37.1141

Rosenkrany, H. S. 939

Rosenthal. A. 1117

Ross. B. 1102

Rossi. F: 1044

Rotem, Y. 1120

Ruiton-Uglicngo, A. I08

Ruscel-Silver dwarfism 885

Rutenberg, W. D. 158

Ruttenberg, H. I). 48
Silck, J. 8.33

Silde, R. M1.1117

Sithn, D. J. 190

Satkiyamat. I. $0+1$

Sillival 827

Salle, B. I. 108. 1180

Santella, R. M. 939

Scarpelli. F. M. 2t

Schärer. K. 1084

Schatub. J. 104.5

Schmicticl. R. 1). 929

Schmidt, H. 1084

Schmidt, R. 1(1)

Schneider. J. A. Al

schott. S. 180

schrocier, W. A. 1102

Schröter, W.

Schulman. I. I). 116.5

Schulte, F. J. 709

Schwart\% H. ('. 1100

Sciatic nerve 24

Sooliosis $8 \times 3$

Seriver. ('. R. 124

Secombe, 1). W. 878

Secular acceleration 695

Secty. J. R. I186

Segal. S. 6\$.

Sell. I: J.916

Sereni, f. g)!

Serum 28.5

Serum calcitonin 7.39

Scrum plasminogen 114

Serum proteins $114 \pi$

Seshia, M. M. K. 276. 74)

secsions, ( $.6+4$

Sevanian. A. 84t.5

Shatic. S. 920

Shaber. R. M. 19

Shatinkin-Kerstenbaum, R. 112

Shani. S. 112

Shapiro, I. J. 894

Shaw. J. C. . . . 10133

Shelton, J. B. 1102

Shelton, J. R. 1102

Sher. J.H. 920

Sherwood. W. ( ; 87t. 1198

Shimada. M. 728

Shin-Buchring. Y. S. 1145

Sickle cell disciase 285

Sicher (O. F.. Jr. 23(1

Sicgel, S. R. 8.37

Singer, A. D. 114

Skiala, J. P. 878

Skeletal muscles 1198

Skeletal tissues 112

sikcleton 920

Skin 862

Skin fibroblasts 76.5

Skin infections 7.32

Skowsky. W. R. 627

Slatopoliky, 1:.7.39

Slv, W. S. 811.816

Small for gestational age (SC A ) infants 108

Smith. (. A. 1080

Smith. 1: 11.33

Simith. F. (i., Jr. 6+4

Smith. J. I). 1180

Simithwick, E : 077

Sodium 153.681

Sodium excretion 1102

Sogatuat. H. 944
Somalomedin 8.8 ?

Somatomatin 83.3 .962

Sordelli, D. (). 1133

Soriano. R. 65

South, M. A.6.3.65.67.71.87

Soukit. I. 1. 783

Speck. W. T. 939. 106.3

spells. (i. 98il

spencer, (;. S. (;. 88s.3

Sperling, D. R. 774

Sperling. M. A.833.962

splecen 64!

Srivatatua, 1. 13

Statal. (j. B. J. 1123

Stafford. H. A. S98

Stahl. P. Sil

Staphlococeus ature'us 11.3 .3

Starling. K. A. 78

Stanvation 793

States, B. 685

Steiner. P. M. 953.957

Stern. R. ( . 977

steroid sulfatase 894

Steroid sulfatase deficiency 894

Stevens, R. I. 894

Stcwart. $11,1+1$

St. (jeme, J. W...Jr. 174

Stocks, J. 1171

Storage discane $8 ! 1,816$

Straus, R. (i. 285

Sturman. J. A. 28

Submaxillary gland 827

Sucrase activity 282

Sulfatide lipidosis 1072

Superoxide dismutase 754

Surfactant 197.770, $8+5$

Sucet. A. Y. Sils

Sucetman. 1. 11+4

Synthetic surfactant 178

Ialafint. E $: 880$

Tallan, H. H. 100

Ian. A. 11145

Ialyi. Q. 796

laturine 28

Iaussig. I.. M. 216. 261

Taylor. (i. R. 67

latylor. J. 1198

lestosterone 7.37

Ietanus 1211

Thaler. M. M. 163.167.171

Theriault, 1.. I.. 758

Thermustability 100

Thibatult, D. W. 119

Thodenius, K. 1109

lhomas, J. M. I $38.11+8$

Ihompson, J. I. 1186

Thymidine kinase 293

Thymidslate synthetase 293

rhymus $7(1) 5$

lierney, D. F. 84t5

Topical decongestants $9(1)$

Total hemolytic activits (CH5) 28.5

Towell, M. 1: 1878

Trace elements $6+6$

Iracer kinction 874

Trachea 45

Iracheobronchial epithelium 977

Iravis. I. B. 33

Travis. S. 1 . 1159 
Trentin. J. J.67.71

Treves. S. 1117

Triglycerides 1.3,19,953

Triiodothyronine 80?

Triiodothyronine ( $\left.\mathrm{T}_{3}\right) 110$

Irygstad, (. W. 959

Tsang. R. (. . 13, 953,957

Isato. F. H. (. $8+9,858$

Tubular myelin 197

Turner. B. M. 86?

Type IV diet 95.3

Type IV hyperlipoproteinemia 953

Tyrosine 6.31

Fyrosine aminotransterase ?

Ultracentrifugation 744

Ultrastructure 197

Umbilical cords 744.871

Undernutrition 29.3

Ureagenesis 303

Uridine kinatse 29.3

Uridyltransferase 1045

Urinary concentration 3.3

Urine 1129

Urman. J. 1033

Urquhart. N. 1192

Vagal control 967

Valine 749
Van Bicrvliet. J. P. (i. M. 1088

Van Biervlict. J. P. M. 112.3

van der Heiden. C. I0ss

vän der Meulen. N. 705

van Haclst. U. 1088

Vatsopressin 627

Vasotocin 627

Ventilatory stability 276

Ventricular epicardial activation ts

Ventricular function 48

Vidnes. 3.943

Visser. I1. K. 1097

Vitamin $B_{t i}$ therapy 100

Vives-Corrons. J. I. 1123

Vogl, T. P. I)6.3

ron Cierke's discalse 936

Wacholder, S. 13

Wadman. S. K. 1088

Walker. P. 116

Wapnir, R. A. 153

Wirshatu. J. B. 754

Water deprivation 33

Weichsel. M. E., Jr. 29.3

Weight 182.701.796

Wennherg. R. P. 16.3.167

West. C. D. 285

Wester. R. C. 7.37

Widdowson. F. M. 1081
Wiesmiann. U. N. 1094

Wilen. R. 701

Willems. J. 1. loss

Williams, 1). M. 823

Williams. M. K. (291

Williamson. A. P. 6.3

Wilson. (;. 13. 139.143,317,986

Wilon. J. B. 1102

Wilson. R. 6.3.65. 67.87

Wilson. S. I. 76,5

Wohl. M. E. B. 252

Wood. D. I. 827

Woodrum. 1). 1: 1051

Wright. R. 186

Yamano. I. 728

Yang. M. (. . 158

Yatuson, (j. 1. 147

Yokoyama, M. 76.5

Zachman. R. I). 849.858

Zeligs, B. J. 197. 1202. 1208

Zeligs. J. D. 197

7.cman. F. J. 97?

Zetterström. R. 1104

Zinc 10.3 .3

Zorab. P. A. 88.3 